REF. TUW 94-10

UGVA-DPT 1994/07-858

\title{
A Renormalized Supersymmetry in the Topological Yang-Mills Field Theory
}

\author{
By A. Brandhuber\#, O. Moritsch执, M.W. de Oliveira据, O. Piguet垉 \\ and M. Schweda \\ \# CERN, CH-1211 Genève 23 (Switzerland) \\ † Département de Physique Théorique, Université de Genève \\ 24, quai Ernest Ansermet, CH-1211 Genève 4 (Switzerland) \\ $\ddagger$ Institut für Theoretische Physik, Technische Universität Wien \\ Wiedner Hauptstraße 8-10, A-1040 Wien (Austria)
}

Abstract. We reconsider the algebraic BRS renormalization of Witten's topological Yang-Mills field theory by making use of a vector supersymmetry Ward identity which improves the finiteness properties of the model. The vector supersymmetric structure is a common feature of several topological theories. The most general local counterterm is determined and is shown to be a trivial BRS-coboundary.

\footnotetext{
${ }^{1}$ Work supported in part by the "Fonds zur Förderung der Wissenschaftlichen Forschung" under Contract Grant Number P9116-PHY.

${ }^{2}$ Work supported in part by the "Fonds zur Förderung der Wissenschaftlichen Forschung", M085-Lise Meitner Fellowship.

${ }^{3}$ Work supported in part by the Swiss National Science Foundation.
} 


\section{Introduction}

The introduction of Witten's topological Yang-Mills field theories in the recent past [1], provided another interesting example of the fruitful interplay between mathematics and physics. With regard to mathematics, these models were devised in order to give a field theoretical interpretation for the Donaldson invariants of four-manifolds [2]. From the point of view of physics, topological field theories could, in principle, give rise to a new class of gravity lagrangians in which the metric tensor would merely play the role of a gauge parameter. Such a remarkable property would essentially be an indication of unbroken general covariance at the quantum level [1, 3].

Mainly for this last reason, the renormalization aspects of Witten's topological model have been widely investigated by several groups in the last few years [4, 5, 6, 7, 8]. In ref. [9], one of the authors discussed the ultraviolet behaviour of the theory in the framework of the algebraic BRS technique [10, 11, 12], which is known to be regularization independent. In particular, one was able to determine, to all orders, the most general local counterterm to the classical action in a Landau type gauge. It was then proven the absence of anomalies and that the cohomological nature of the model was totally insensitive to quantum effects.

In the present paper, we will be concerned in extending the analysis of ref. [9], by exploiting an additional invariance of the theory: namely topological vector supersymmetry which together with BRS-symmetry and translations build a supersymmetry algebra of Wess-Zumino type [14, 13. This supersymmetric structure, whose topological origin is manifest when a Landau gauge is chosen, is a common feature of a large class of topological models [13, 15, 16]. One will realize that the associated supersymmetric Ward identity significantly improves, with respect to [9], the finiteness properties of the theory, restraining the number of monomials in the fields which show up in the counterterm expression. It is worthwhile to underline once more that our approach does not make reference to any regularization scheme and extends to all orders of perturbation theory.

The work is organized as follows: in Section 2 we describe the particular Landau gauge-fixing in the classical approximation and its quantization, in Section 3 we show the absence of anomalies in the theory and we obtain the local counterterm. Section 4 contains some concluding remarks. We also devote two appendices to a better understanding of the counterterm construction.

\section{The Classical Action and the Landau Gauge}

Let us start by presenting the BRS framework of Witten's topological Yang-Mills field theory in $D=4$ Euclidean space. We adopt here the standard construction of refs. 17, 18, 19] and introduce the following set of fields: a gauge connection $A_{\mu}^{a}$, an anticommuting vector field $\psi_{\mu}^{a}$ (also called topological ghost), a pair of antiself-dual tensor fields $\left(B_{\mu \nu}^{a}, \chi_{\mu \nu}^{a}\right)$ and two ghost fields $\left(c^{a}, \varphi^{a}\right)$. One needs also a couple of Lagrange multipliers $\left(b^{a}, \eta^{a}\right)$ and a couple of antighosts $\left(\bar{c}^{a}, \bar{\varphi}^{a}\right)$. These variables will take values in the adjoint representation 
of an arbitrary compact gauge group $\mathbf{G}$ with structure constants $f^{a b c}$. We give their respective dimensions and Faddeev-Popov ghost charges $(\Phi \Pi)$ in Table 1:

\begin{tabular}{|c|c|c|c|c|c|c|c|c|c|c|}
\hline & $A_{\mu}$ & $\psi_{\mu}$ & $c$ & $\varphi$ & $\chi_{\mu \nu}$ & $B_{\mu \nu}$ & $\bar{\varphi}$ & $\eta$ & $\bar{c}$ & $b$ \\
\hline dimension & 1 & 1 & 0 & 0 & 2 & 2 & 2 & 2 & 2 & 2 \\
\hline$\Phi \Pi$ & 0 & 1 & 1 & 2 & -1 & 0 & -2 & -1 & -1 & 0 \\
\hline
\end{tabular}

Table 1: Dimensions and Faddeev-Popov ghost charges of the fields.

The nilpotent BRS transformations for the fields defining the model were originally obtained by Baulieu and Singer [17] (see also [18]) and read as:

$$
\begin{array}{ll}
s A_{\mu}^{a}=-\left(D_{\mu} c\right)^{a}+\psi_{\mu}^{a}, & \\
s \psi_{\mu}^{a}=f^{a b c} c^{b} \psi_{\mu}^{c}+\left(D_{\mu} \varphi\right)^{a}, & \\
s \varphi^{a}=f^{a b c} c^{b} \varphi^{c}, & s B_{\mu \nu}^{a}=0, \\
s c^{a}=\frac{1}{2} f^{a b c} c^{b} c^{c}+\varphi^{a}, & s \eta^{a}=0, \\
s \chi_{\mu \nu}^{a}=B_{\mu \nu}^{a}, & s b^{a}=0 . \\
s \bar{\varphi}^{a}=\eta^{a}, &
\end{array}
$$

As one can also infer from ref. [17], in Euclidean flat space Witten's classical action takes the form of a pure gauge fixing term which enforces the topological properties of the (anti-)instantonic field configurations of $A_{\mu}^{a}$. In the present work we propose the following Landau type gauge-fixed action:

$$
\begin{aligned}
S_{\mathrm{gf}}=s \int d^{4} x & {\left[\chi^{a \mu \nu} F_{\mu \nu}^{+a}+\bar{\varphi}^{a}\left(\partial_{\mu} \psi^{\mu}\right)^{a}+\bar{c}^{a} \partial_{\mu} A^{a \mu}\right]=} \\
=\int d^{4} x & {\left[B^{a \mu \nu} F_{\mu \nu}^{+a}-\chi^{a \mu \nu}\left(\delta_{\mu}^{\rho} \delta_{\nu}^{\sigma}+\frac{1}{2} \varepsilon_{\mu \nu}^{\rho \sigma}\right)\left(D_{\rho} \psi_{\sigma}\right)^{a}+\chi^{a \mu \nu} f^{a b c} F_{\mu \nu}^{+b} c^{c}\right.} \\
& +\eta^{a} \partial^{\mu} \psi_{\mu}^{a}+\bar{\varphi}^{a} \partial^{\mu}\left(D_{\mu} \varphi\right)^{a}+\bar{\varphi}^{a} \partial^{\mu}\left(f^{a b c} c^{b} \psi_{\mu}^{c}\right)+b^{a} \partial^{\mu} A_{\mu}^{a} \\
& \left.+\bar{c}^{a} \partial^{\mu}\left(D_{\mu} c\right)^{a}-\bar{c}^{a} \partial^{\mu} \psi_{\mu}^{a}\right]
\end{aligned}
$$

where

$$
F_{\mu \nu}^{+a}=\frac{1}{2}\left(F_{\mu \nu}^{a}+\widetilde{F}_{\mu \nu}^{a}\right)
$$

stands for the antiself-dual part of the Yang-Mills field strength with

$$
\widetilde{F}_{\mu \nu}^{a}=\frac{1}{2} \varepsilon_{\mu \nu}{ }^{\rho \sigma} F_{\rho \sigma}^{a} .
$$


It should be noticed however that $S_{\text {gf }}$ displays a significant difference with respect to the original and well-known action for the topological gauge field theory «17, 18, 4, 9]: the gauge choice imposed on the topological ghost makes use of the ordinary space-time partial derivative, viz.,

$$
\partial_{\mu} \psi^{a \mu}=0,
$$

instead of the gauge-covariant one. One will see shortly that the main motivation for such a modification relies on the fact that the action (2.2) possesses a larger content of symmetries, in particular supersymmetry, than that of the covariant situation [9]. We also stress that condition (2.5) is as acceptable as any other choice of gauge condition since a different one should not modify the physical output. Indeed, in this work one is mainly concerned with a better understanding of the ultraviolet behaviour of the model. The possible consequences to the structure of the topological observables [1] that may eventually be produced by (2.5) are not analyzed here.

In order to translate the BRS invariance of the gauge-fixed action (2.2) into a Slavnov identity, one follows a general rule [11], coupling the non-linear parts of the transformations (2.1) to a set of external sources $\left(\Omega^{a \mu}, \tau^{a \mu}, L^{a}, D^{a}\right)$. Their dimensions and FaddeevPopov charges are defined in Table 2:

\begin{tabular}{|c|c|c|c|c|}
\hline & $\Omega^{\mu}$ & $\tau^{\mu}$ & $L$ & $D$ \\
\hline dimension & 3 & 3 & 4 & 4 \\
\hline$\Phi \Pi$ & -1 & -2 & -2 & -3 \\
\hline
\end{tabular}

Table 2: Dimensions and Faddeev-Popov ghost charges of the external sources.

The invariant external action is then chosen to be:

$$
S_{\mathrm{ext}}=\int d^{4} x\left[\Omega^{a \mu}\left(D_{\mu} c\right)^{a}+\tau^{a \mu}\left(s \psi_{\mu}^{a}\right)+\frac{1}{2} L^{a} f^{a b c} c^{b} c^{c}+D^{a}\left(s \varphi^{a}\right)\right],
$$

where one imposes a BRS-doublet structure for the transformation laws of the sources,

$$
\begin{aligned}
& s \tau_{\mu}^{a}=\Omega_{\mu}^{a}, \quad s \Omega_{\mu}^{a}=0, \\
& s D^{a}=L^{a}, \quad s L^{a}=0 .
\end{aligned}
$$

The complete action is then given by an exact BRS-variation:

$$
\begin{aligned}
\Sigma & =S_{\mathrm{gf}}+S_{\mathrm{ext}}= \\
& =s \int d^{4} x\left[\chi^{a \mu \nu} F_{\mu \nu}^{+a}+\bar{\varphi}^{a} \partial_{\mu} \psi^{a \mu}+\bar{c}^{a} \partial_{\mu} A^{a \mu}+\tau^{a \mu}\left(D_{\mu} c\right)^{a}+\frac{1}{2} D^{a} f^{a b c} c^{b} c^{c}\right],
\end{aligned}
$$

and satisfies the Slavnov identity:

$$
\mathcal{S}(\Sigma)=0
$$


with,

$$
\begin{aligned}
\mathcal{S}(\Sigma)=\int d^{4} x & \left(\psi_{\mu}^{a} \frac{\delta \Sigma}{\delta A_{\mu}^{a}}-\frac{\delta \Sigma}{\delta \Omega^{a \mu}} \frac{\delta \Sigma}{\delta A_{\mu}^{a}}+\varphi^{a} \frac{\delta \Sigma}{\delta c^{a}}+\frac{\delta \Sigma}{\delta L^{a}} \frac{\delta \Sigma}{\delta c^{a}}+\frac{\delta \Sigma}{\delta \tau^{a \mu}} \frac{\delta \Sigma}{\delta \psi_{\mu}^{a}}+\frac{\delta \Sigma}{\delta D^{a}} \frac{\delta \Sigma}{\delta \varphi^{a}}\right. \\
& \left.+\frac{1}{2} B_{\mu \nu}^{a} \frac{\delta \Sigma}{\delta \chi_{\mu \nu}^{a}}+\eta^{a} \frac{\delta \Sigma}{\delta \bar{\varphi}^{a}}+b^{a} \frac{\delta \Sigma}{\delta \bar{c}^{a}}+\Omega_{\mu}^{a} \frac{\delta \Sigma}{\delta \tau_{\mu}^{a}}+L^{a} \frac{\delta \Sigma}{\delta D^{a}}\right)
\end{aligned}
$$

From the Slavnov identity one reads off the linearized BRS operator:

$$
\begin{aligned}
\mathcal{B}_{\Sigma}=\int d^{4} x & \left(\psi_{\mu}^{a} \frac{\delta}{\delta A_{\mu}^{a}}-\frac{\delta \Sigma}{\delta \Omega^{a \mu}} \frac{\delta}{\delta A_{\mu}^{a}}-\frac{\delta \Sigma}{\delta A_{\mu}^{a}} \frac{\delta}{\delta \Omega^{a \mu}}+\varphi^{a} \frac{\delta}{\delta c^{a}}+\frac{\delta \Sigma}{\delta L^{a}} \frac{\delta}{\delta c^{a}}+\frac{\delta \Sigma}{\delta c^{a}} \frac{\delta}{\delta L^{a}}\right. \\
& +\frac{\delta \Sigma}{\delta \tau^{a \mu}} \frac{\delta}{\delta \psi_{\mu}^{a}}+\frac{\delta \Sigma}{\delta \psi_{\mu}^{a}} \frac{\delta}{\delta \tau^{a \mu}}+\frac{\delta \Sigma}{\delta D^{a}} \frac{\delta}{\delta \varphi^{a}}+\frac{\delta \Sigma}{\delta \varphi^{a}} \frac{\delta}{\delta D^{a}}+\frac{1}{2} B_{\mu \nu}^{a} \frac{\delta}{\delta \chi_{\mu \nu}^{a}} \\
& \left.+\eta^{a} \frac{\delta}{\delta \bar{\varphi}^{a}}+b^{a} \frac{\delta}{\delta \bar{c}^{a}}+\Omega_{\mu}^{a} \frac{\delta}{\delta \tau_{\mu}^{a}}+L^{a} \frac{\delta}{\delta D^{a}}\right)
\end{aligned}
$$

Let us remark that it is a nilpotent operator:

$$
\mathcal{B}_{\Sigma} \mathcal{B}_{\Sigma}=0
$$

The Landau gauge-fixing conditions are:

$$
\frac{\delta \Sigma}{\delta b^{a}}=\partial^{\mu} A_{\mu}^{a}
$$

and

$$
\frac{\delta \Sigma}{\delta \eta^{a}}=\partial^{\mu} \psi_{\mu}^{a}
$$

As usual [12, by (anti)commuting the gauge conditions (2.13)-(2.14) with the Slavnov identity (2.9), one gets two ghost equations:

$$
\frac{\delta \Sigma}{\delta \bar{c}^{a}}-\partial^{\mu} \frac{\delta \Sigma}{\delta \Omega^{a \mu}}=-\partial^{\mu} \psi_{\mu}^{a},
$$

and

$$
\frac{\delta \Sigma}{\delta \bar{\varphi}^{a}}-\partial^{\mu} \frac{\delta \Sigma}{\delta \tau^{a \mu}}=0
$$

The complete action $\Sigma$ obeys, in addition, a set of global constraints. They are derived by making use of the algebraic structure of the Landau gauge-fixings imposed on the fields $A_{\mu}^{a}$ and $\psi_{\mu}^{a}$. The first one is an antighost equation [20], which controls the coupling of the ghost $\varphi$ :

$$
\mathcal{G}^{a} \Sigma=\Delta_{\mathcal{G}}^{a}
$$

where,

$$
\mathcal{G}^{a}=\int d^{4} x\left(\frac{\delta}{\delta \varphi^{a}}-f^{a b c} \bar{\varphi}^{b} \frac{\delta}{\delta b^{c}}\right)
$$

and

$$
\Delta_{\mathcal{G}}^{a}=\int d^{4} x f^{a b c}\left(\tau^{b \mu} A_{\mu}^{c}+D^{b} c^{c}\right)
$$


We remark that $\Delta_{\mathcal{G}}^{a}$ is a classical breaking, for it is linear in the quantum fields. This means that it can be promoted to the full quantum theory without specific renormalization.

Commuting the antighost equation (2.17) with the Slavnov identity (2.9), one obtains a further condition on $\Sigma$ (again classically broken):

$$
\mathcal{F}^{a} \Sigma=\Delta_{\mathcal{F}}^{a}
$$

with

$$
\begin{aligned}
\mathcal{F}^{a}=\int d^{4} x & \left(\frac{\delta}{\delta c^{a}}-f^{a b c} \bar{\varphi}^{b} \frac{\delta}{\delta \bar{c}^{c}}-f^{a b c} A_{\mu}^{b} \frac{\delta}{\delta \psi_{\mu}^{c}}-f^{a b c} \tau_{\mu}^{b} \frac{\delta}{\delta \Omega_{\mu}^{c}}\right. \\
& \left.-f^{a b c} c^{b} \frac{\delta}{\delta \varphi^{c}}-f^{a b c} D^{b} \frac{\delta}{\delta L^{c}}+f^{a b c} \eta^{b} \frac{\delta}{\delta b^{c}}\right)
\end{aligned}
$$

and

$$
\Delta_{\mathcal{F}}^{a}=\int d^{4} x f^{a b c}\left(D^{b} \varphi^{c}-\Omega^{b \mu} A_{\mu}^{c}-\tau^{b \mu} \psi_{\mu}^{c}-L^{b} c^{c}\right)
$$

Moreover, there exists a second antighost equation, controlling the coupling of the ghost $c^{a}$ :

$$
\mathcal{G}^{\prime a} \Sigma=\Delta_{\mathcal{G}^{\prime}}^{a}
$$

where,

$$
\mathcal{G}^{\prime a}=\int d^{4} x\left(\frac{\delta}{\delta c^{a}}+\frac{1}{2} f^{a b c} \chi^{b \mu \nu} \frac{\delta}{\delta B^{c \mu \nu}}+f^{a b c} \bar{\varphi}^{b} \frac{\delta}{\delta \eta^{c}}+f^{a b c} \bar{c}^{b} \frac{\delta}{\delta b^{c}}\right)
$$

and

$$
\Delta_{\mathcal{G}^{\prime}}^{a}=\int d^{4} x f^{a b c}\left(D^{b} \varphi^{c}-\Omega^{b \mu} A_{\mu}^{c}-\tau^{b \mu} \psi_{\mu}^{c}-L^{b} c^{c}\right)
$$

By anticommuting (2.23) with (2.9) one sees that $\Sigma$ is left invariant under the rigid gauge symmetry of the theory:

$$
\mathcal{R}^{a} \Sigma=0,
$$

with

$$
\mathcal{R}^{a}=\sum_{\text {all fields } \Phi} \int d^{4} x f^{a b c} \Phi^{b} \frac{\delta}{\delta \Phi^{c}}
$$


As announced in the introduction, the complete action $\Sigma$ exhibits, besides the BRS invariance (2.1), an ulterior global symmetry, the topological vector supersymmetry, whose generators $\delta_{\alpha}$ bear a Lorentz index. Its action on the fields and sources is:

$$
\begin{array}{ll}
\delta_{\alpha} A_{\mu}^{a}=0, & \delta_{\alpha} \psi_{\mu}^{a}=\partial_{\alpha} A_{\mu}^{a}, \\
\delta_{\alpha} c^{a}=0, & \delta_{\alpha} \varphi^{a}=\partial_{\alpha} c^{a}, \\
\delta_{\alpha} \chi_{\mu \nu}^{a}=0, & \delta_{\alpha} B_{\mu \nu}^{a}=\partial_{\alpha} \chi_{\mu \nu}^{a}, \\
\delta_{\alpha} \bar{\varphi}^{a}=0, & \delta_{\alpha} \eta^{a}=\partial_{\alpha} \bar{\varphi}^{a}, \\
\delta_{\alpha} \bar{c}^{a}=\partial_{\alpha} \bar{\varphi}^{a}, & \delta_{\alpha} b^{a}=\partial_{\alpha} \bar{c}^{a}-\partial_{\alpha} \eta^{a}, \\
\delta_{\alpha} \tau_{\mu}^{a}=0, & \delta_{\alpha} \Omega_{\mu}^{a}=\partial_{\alpha} \tau_{\mu}^{a}, \\
\delta_{\alpha} D^{a}=0, & \delta_{\alpha} L^{a}=\partial_{\alpha} D^{a} .
\end{array}
$$

The generators $\delta_{\alpha}$ satisfy, together with the BRS operator, a supersymmetry algebra:

$$
\left\{s, \delta_{\alpha}\right\}=\partial_{\alpha} \text {. }
$$

Let us note, that this algebra is automatically valid off-shell. The associated supersymmetry Ward identity writes

$$
\mathcal{W}_{\alpha} \Sigma=0
$$

where

$$
\begin{aligned}
\mathcal{W}_{\alpha}=\int d^{4} x & {\left[\left(\partial_{\alpha} A_{\mu}^{a}\right) \frac{\delta}{\delta \psi_{\mu}^{a}}+\left(\partial_{\alpha} c^{a}\right) \frac{\delta}{\delta \varphi^{a}}+\frac{1}{2}\left(\partial_{\alpha} \chi_{\mu \nu}^{a}\right) \frac{\delta}{\delta B_{\mu \nu}^{a}}+\left(\partial_{\alpha} \bar{\varphi}^{a}\right) \frac{\delta}{\delta \eta^{a}}\right.} \\
& \left.+\left(\partial_{\alpha} \bar{c}^{a}-\partial_{\alpha} \eta^{a}\right) \frac{\delta}{\delta b^{a}}+\left(\partial_{\alpha} \bar{\varphi}^{a}\right) \frac{\delta}{\delta \bar{c}^{a}}+\left(\partial_{\alpha} \tau_{\mu}^{a}\right) \frac{\delta}{\delta \Omega_{\mu}^{a}}+\left(\partial_{\alpha} D^{a}\right) \frac{\delta}{\delta L^{a}}\right] .
\end{aligned}
$$

We summarize the above results: the complete action $\Sigma$ in (2.8) fulfills

(i) the Slavnov identity

$$
\mathcal{S}(\Sigma)=0
$$

(ii) the two gauge-fixing conditions, eqs.(2.13)-(2.14),

(iii) the two ghost equations, eqs.2.15)-(2.16),

(iv) the first antighost equation

$$
\mathcal{G}^{a} \Sigma=\Delta_{\mathcal{G}}^{a}
$$

(v) the $\mathcal{F}$-Ward identity

$$
\mathcal{F}^{a} \Sigma=\Delta_{\mathcal{F}}^{a}
$$


(vi) the second antighost equation

$$
\mathcal{G}^{\prime a} \Sigma=\Delta_{\mathcal{G}^{\prime}}^{a}
$$

(vii) the rigid invariance

$$
\mathcal{R}^{a} \Sigma=0
$$

and,

(viii) the global supersymmetry Ward identity

$$
\mathcal{W}_{\alpha} \Sigma=0
$$

We close this section by displaying the graded Lie algebra obeyed by the functional operators $\mathcal{B}_{\Sigma}, \mathcal{G}^{a}, \mathcal{F}^{a}, \mathcal{G}^{\prime a}, \mathcal{R}^{a}$ and $\mathcal{W}_{\alpha}$ :

$$
\left\{\mathcal{B}_{\Sigma}, \mathcal{B}_{\Sigma}\right\}=0
$$

$$
\begin{aligned}
& {\left[\mathcal{G}^{a}, \mathcal{B}_{\Sigma}\right]=\mathcal{F}^{a},} \\
& \left\{\mathcal{F}^{a}, \mathcal{B}_{\Sigma}\right\}=0 \\
& {\left[\mathcal{G}^{a}, \mathcal{G}^{b}\right]=0} \\
& {\left[\mathcal{G}^{a}, \mathcal{F}^{b}\right]=0,} \\
& \left\{\mathcal{F}^{a}, \mathcal{F}^{b}\right\}=0 \\
& \left\{\mathcal{G}^{\prime a}, \mathcal{G}^{\prime b}\right\}=0 \\
& \left\{\mathcal{G}^{\prime a}, \mathcal{B}_{\Sigma}\right\}=\mathcal{R}^{a}, \\
& {\left[\mathcal{R}^{a}, \mathcal{B}_{\Sigma}\right]=0} \\
& {\left[\mathcal{R}^{a}, \mathcal{G}^{\prime b}\right]=-f^{a b c} \mathcal{G}^{\prime c},} \\
& {\left[\mathcal{R}^{a}, \mathcal{R}^{b}\right]=-f^{a b c} \mathcal{R}^{c},} \\
& {\left[\mathcal{G}^{a}, \mathcal{G}^{\prime b}\right]=0,} \\
& \left\{\mathcal{F}^{a}, \mathcal{G}^{\prime b}\right\}=-f^{a b c} \mathcal{G}^{c}, \\
& {\left[\mathcal{R}^{a}, \mathcal{G}^{b}\right]=-f^{a b c} \mathcal{G}^{c},} \\
& {\left[\mathcal{R}^{a}, \mathcal{F}^{b}\right]=-f^{a b c} \mathcal{F}^{c},} \\
& \left\{\mathcal{W}_{\alpha}, \mathcal{B}_{\Sigma}\right\}=\mathcal{P}_{\alpha} \\
& \left\{\mathcal{W}_{\alpha}, \mathcal{W}_{\beta}\right\}=0 \\
& {\left[\mathcal{G}^{a}, \mathcal{W}_{\alpha}\right]=0} \\
& \left\{\mathcal{F}^{a}, \mathcal{W}_{\alpha}\right\}=0 \\
& \left\{\mathcal{G}^{\prime a}, \mathcal{W}_{\alpha}\right\}=0 \\
& {\left[\mathcal{R}^{a}, \mathcal{W}_{\alpha}\right]=0}
\end{aligned}
$$


where $\mathcal{P}_{\alpha}$ denotes the Ward operator for translations in the space of fields,

$$
\mathcal{P}_{\alpha}=\sum_{\text {all fields } \Phi} \int d^{4} x\left(\partial_{\alpha} \Phi\right) \frac{\delta}{\delta \Phi} .
$$

\section{BRS Cohomology: Anomalies and Counterterms}

The purpose of this section is to present a systematic procedure for the evaluation of the possible local counterterms to the complete action $\Sigma$ in (2.8). One has to observe that the structure of any quantum correction will be entirely governed by the set of classical constraints on $\Sigma$, as long as they can be extended to the quantum level. This latter statement implies that the absence of Slavnov and Ward identity anomalies has to be demonstrated previously.

With this aim, we adopt the general recipe [21] of collecting all the symmetry operators derived in the last section into a unique functional operator which is nilpotent by construction. It turns out then that the discussion of the renormalization properties of the model is significantly simplified when this strategy is implemented. The first step consists of the introduction of three pairs of global ghosts $\left(\xi^{\mu}, \theta^{\mu}\right),\left(u^{a}, v^{a}\right)$ and $\left(w^{a}, y^{a}\right)$ with the following dimensions and $\Phi \Pi$ assignments:

\begin{tabular}{|c|c|c|c|c|c|c|}
\hline & $\xi$ & $\theta$ & $\mathrm{u}$ & $\mathrm{v}$ & $\mathrm{w}$ & $\mathrm{y}$ \\
\hline dimension & -1 & -1 & 0 & 0 & 0 & 0 \\
\hline$\Phi \Pi$ & 2 & 1 & 3 & 2 & 2 & 1 \\
\hline
\end{tabular}

Table 3: Dimensions and Faddeev-Popov charges of the global ghosts.

With the help of these ghosts one builds up the operator $\delta$ as below:

$$
\begin{aligned}
\delta= & \mathcal{B}_{\Sigma}+\xi^{\alpha} \mathcal{W}_{\alpha}+\theta^{\alpha} \mathcal{P}_{\alpha}+u^{a} \mathcal{G}^{a}+v^{a} \mathcal{F}^{a}+w^{a} \mathcal{G}^{\prime a}+y^{a} \mathcal{R}^{a}-\xi^{\mu} \frac{\partial}{\partial \theta^{\mu}} \\
& -u^{a} \frac{\partial}{\partial v^{a}}-w^{a} \frac{\partial}{\partial y^{a}}+f^{a b c} y^{a} u^{b} \frac{\partial}{\partial u^{c}}+f^{a b c} y^{a} v^{b} \frac{\partial}{\partial v^{c}} \\
& +f^{a b c} y^{a} w^{b} \frac{\partial}{\partial w^{c}}+\frac{1}{2} f^{a b c} y^{a} y^{b} \frac{\partial}{\partial y^{c}}-f^{a b c} w^{a} v^{b} \frac{\partial}{\partial u^{c}} .
\end{aligned}
$$

One easily verifies that $\delta$ is nilpotent:

$$
\delta \delta=0
$$

It should be clear at this stage that all the relevant features of the linear algebra (2.38) are encoded in the extended operator $\delta$. As a consequence, the analysis of possible anomalies and allowed counterterms reduces to the study of the BRS-cohomology $\mathcal{H}^{*}(\delta)$

of $\delta$ in the sectors of local polynomials in the fields and sources characterized by the proper ghost charge. 
More specifically, the two distinct sectors one has to consider are:

- Anomalies:

- cohomology of $\delta$ in the sector of local integrated polynomials of dimension four and one unit of Faddeev-Popov ghost charge

$$
\delta \mathcal{A}=0, \quad \mathcal{A} \neq \delta \hat{\mathcal{A}} \text { and } \Phi \Pi(\mathcal{A})=1,
$$

- Counterterms:

- cohomology of $\delta$ in the sector of local integrated polynomials of dimension four and Faddeev-Popov ghost charge zero

$$
\delta \widetilde{\Sigma}=0, \quad \widetilde{\Sigma} \neq \delta \Delta \text { and } \Phi \Pi(\widetilde{\Sigma})=0
$$

where $\widetilde{\Sigma}$ is independent of the global ghosts $(\xi, \theta, \mathrm{u}, \mathrm{v}, \mathrm{w}, \mathrm{y})$.

Now, to characterize the cohomology of $\delta$ we define a filtration operator $\mathcal{N}$ as follows:

$$
\begin{aligned}
\mathcal{N}= & 2 \xi^{\mu} \frac{\partial}{\partial \xi^{\mu}}+2 \theta^{\mu} \frac{\partial}{\partial \theta^{\mu}}+2 u^{a} \frac{\partial}{\partial u^{a}}+2 v^{a} \frac{\partial}{\partial v^{a}}+2 w^{a} \frac{\partial}{\partial w^{a}}+2 y^{a} \frac{\partial}{\partial y^{a}} \\
& +\int d^{4} x\left[c^{a} \frac{\delta}{\delta c^{a}}+\varphi^{a} \frac{\delta}{\delta \varphi^{a}}+2\left(\frac{1}{2} B_{\mu \nu}^{a} \frac{\delta}{\delta B_{\mu \nu}^{a}}\right)+2\left(\frac{1}{2} \chi_{\mu \nu}^{a} \frac{\delta}{\delta \chi_{\mu \nu}^{a}}\right)\right. \\
& \left.+2 \eta^{a} \frac{\delta}{\delta \eta^{a}}+2 \bar{\varphi}^{a} \frac{\delta}{\delta \bar{\varphi}^{a}}+2 b^{a} \frac{\delta}{\delta b^{a}}+2 \bar{c}^{a} \frac{\delta}{\delta \bar{c}^{a}}+\Omega_{\mu}^{a} \frac{\delta}{\delta \Omega_{\mu}^{a}}+\tau_{\mu}^{a} \frac{\delta}{\delta \tau_{\mu}^{a}}\right] .
\end{aligned}
$$

By means of a simple inspection, one notices that $\mathcal{N}$ has the structure of a counting operator which clearly induces a separation on $\delta$, namely,

$$
\delta=\sum_{n=0}^{\bar{n}} \delta^{(n)}
$$

with,

$$
\left[\mathcal{N}, \delta^{(n)}\right]=n \delta^{(n)}
$$

We present here the explicit expression for $\delta^{(0)}$ :

$$
\begin{aligned}
\delta^{(0)}= & \int d^{4} x\left(\psi_{\mu}^{a} \frac{\delta}{\delta A_{\mu}^{a}}+\varphi^{a} \frac{\delta}{\delta c^{a}}+\frac{1}{2} B_{\mu \nu}^{a} \frac{\delta}{\delta \chi_{\mu \nu}^{a}}+\eta^{a} \frac{\delta}{\delta \bar{\varphi}^{a}}+b^{a} \frac{\delta}{\delta \bar{c}^{a}}+\Omega_{\mu}^{a} \frac{\delta}{\delta \tau_{\mu}^{a}}+L^{a} \frac{\delta}{\delta D^{a}}\right) \\
& -\xi^{\mu} \frac{\partial}{\partial \theta^{\mu}}-u^{a} \frac{\partial}{\partial v^{a}}-w^{a} \frac{\partial}{\partial y^{a}}
\end{aligned}
$$

and we remark that $\delta^{(0)}$ is also nilpotent as a direct consequence of eq.(3.2).

One immediately notices a remarkable aspect about $\delta^{(0)}$ : all fields, sources and global ghosts appear in BRS-doublets. This implies [22, 23, 24] that the cohomology of $\delta^{(0)}$, acting on the unconstrained space of integrated local field polynomials independent of the global ghosts is empty, i.e.

$$
\mathcal{H}^{*}\left(\delta^{(0)}\right)=\emptyset
$$


Hence, the cohomology of $\delta$ vanishes as well, in view of the fact that $\mathcal{H}^{*}(\delta)$ is isomorphic to a subspace of $\mathcal{H}^{*}\left(\delta^{(0)}\right)$. This last consequence relies on a very general derivation developed in [22] by using the method of spectral sequences. Therefore we can conclude:

$$
\mathcal{H}^{*}(\delta)=\emptyset .
$$

This result implies that the Slavnov identity (2.9) is non-anomalous as well as the whole set of Ward identities (2.17), (2.20), (2.23), (2.26) and (2.30). This will mean that, besides the antighost equations, also the vector supersymmetry Ward identity (2.30) can be employed as a stability constraint for selecting invariant counterterms.

Let us now turn to the computation of the invariant counterterms. The result (3.10) already implies that they are $\delta$ variations, but this is not enough for our purpose because we have to discard those which depend on the global ghosts. A way out is to study the cohomology of $\mathcal{B}_{\Sigma}$ and then to impose the other Ward identities as constraints.

We first remark that the cohomology of $\mathcal{B}_{\Sigma}$ in the space of local functionals depending on all the fields (but without the global ghosts) is empty, i.e.:

$$
\mathcal{H}^{*}\left(\mathcal{B}_{\Sigma}\right)=\emptyset .
$$

The proof is immediate [9]. We first observe that the operator $\mathcal{N}$ (3.5) (with the global ghosts set to zero) constitutes a filtration of the operator $\mathcal{B}_{\Sigma}$, with $\mathcal{B}_{\Sigma}^{(0)}$ coinciding with $\delta^{(0)}$ (3.8) (global ghosts set to zero, too). Than the proof follows from the triviality of $\delta^{(0)}$.

Since the general counterterm $\widetilde{\Sigma}$ has to obey the BRS invariance constraint

$$
\mathcal{B}_{\Sigma} \widetilde{\Sigma}=0,
$$

we thus know that it is the $\mathcal{B}_{\Sigma}$-variation of some local functional $\Delta$ of dimension 4 and ghost charge -1 ,

$$
\widetilde{\Sigma}=\mathcal{B}_{\Sigma} \Delta .
$$

Recall now that $\widetilde{\Sigma}$ is a local integrated polynomial in the fields and sources with dimension four and ghost charge zero. It is required to obey the following constraints:

$$
\begin{gathered}
\mathcal{W}_{\alpha} \widetilde{\Sigma}=0, \quad \mathcal{P}_{\alpha} \widetilde{\Sigma}=0, \\
\mathcal{G}^{a} \widetilde{\Sigma}=0, \quad \mathcal{F}^{a} \widetilde{\Sigma}=0, \quad \mathcal{G}^{\prime a} \widetilde{\Sigma}=0, \quad \mathcal{R}^{a} \widetilde{\Sigma}=0, \\
\frac{\delta \widetilde{\Sigma}}{\delta \eta^{a}}=0, \quad \frac{\delta \widetilde{\Sigma}}{\delta b^{a}}=0, \quad \frac{\delta \widetilde{\Sigma}}{\delta \bar{\varphi}^{a}}-\partial^{\mu} \frac{\delta \widetilde{\Sigma}}{\delta \tau^{a \mu}}=0, \frac{\delta \widetilde{\Sigma}}{\delta \bar{c}^{a}}-\partial^{\mu} \frac{\delta \widetilde{\Sigma}}{\delta \Omega^{a \mu}}=0 .
\end{gathered}
$$

Let us here make two comments: Firstly, one has to notice that all possible quantum corrections to $\Sigma$ are given by BRS-coboundaries: this assures that the BRS-triviality of the classical action is preserved at the quantum level. Secondly, since there are no 
physical parameters in the model, one is essentially interested in the study of anomalous dimensions, these latters being related to BRS-trivial counterterms.

$\widetilde{\Sigma}$ obeying the constraints above can we choose $\Delta$ to obey them, too ? It is clear that, since the set of constraints above is stable under the action of $\mathcal{B}_{\Sigma}$ (see (2.38)), $\widetilde{\Sigma}$ will obey these constraints if $\Delta$ does. Although $\Delta$ does not have to fulfill them it may be chosen to do so, except for the constraint given by the third eq. of (3.15). This is shown in Appendix A for the conditions given by the fourth eq. of (3.15) and by eqs. (3.16) and in Appendix B for the first two of (3.15). To summarize we can write

$$
\mathcal{G}^{a} \Delta=\mathcal{F}^{a} \Delta=\mathcal{R}^{a} \Delta=\frac{\delta \Delta}{\delta \eta^{a}}=\frac{\delta \Delta}{\delta b^{a}}=\frac{\delta \Delta}{\delta \bar{\varphi}^{a}}-\partial^{\mu} \frac{\delta \Delta}{\delta \tau^{a \mu}}=\frac{\delta \Delta}{\delta \bar{c}^{a}}-\partial^{\mu} \frac{\delta \Delta}{\delta \Omega^{a \mu}}=0 .
$$

This gives rise to a rather small set of independent counterterms $\widetilde{\Sigma}$. This set is further reduced by applying the last constraints, namely the third of eqs. (3.15) and, last but not least, the supersymmetry constraint (3.14) (translation invariance is obvious).

Finally one is able to express the most general local conterterm $\widetilde{\Sigma}$ as:

$$
\widetilde{\Sigma}=\widetilde{\Sigma}^{(2)}+\widetilde{\Sigma}^{(3)}
$$

with,

$$
\begin{aligned}
\widetilde{\Sigma}^{(2)}=\mathcal{B}_{\Sigma} \int & d^{4} x\left\{a_{1}\left[\left(\Omega^{a \mu}-\partial^{\mu} \bar{c}^{a}\right) A_{\mu}^{a}+\left(\tau^{a \mu}-\partial^{\mu} \bar{\varphi}^{a}\right) \psi_{\mu}^{a}\right]\right. \\
& \left.+a_{2}\left(\tau^{a \mu}-\partial^{\mu} \bar{\varphi}^{a}\right) \partial_{\mu} c^{a}+a_{3} \chi^{a \mu \nu} \partial_{\mu} A_{\nu}^{a}\right\}
\end{aligned}
$$

and

$$
\widetilde{\Sigma}^{(3)}=\mathcal{B}_{\Sigma} \int d^{4} x\left\{b f^{a b c} \chi^{a \mu \nu} A_{\mu}^{b} A_{\nu}^{c}\right\}
$$

where $a_{1}, a_{2}, a_{3}$ and $b$ are the arbitrary coefficients of the four possible counterterms. We emphasize that no other combination of fields and sources would be compatible with eqs.(3.12),(3.14)-(3.16).

An interesting result of the present study is that also with the partial-derivative Landau type gauge used here (2.5), the counterterm

$$
\int d^{4} x\left(F_{\mu \nu}^{+}\right)^{2}
$$

does not show up. This agrees with [9] and with a previous 1-loop computation carried out in [7, 3].

\section{Concluding Remarks}

In this paper we have investigated the issue of the renormalization of Witten's topological Yang-Mills field theory in the presence of several invariances. In particular, it has been 
made direct use of a vector supersymmetric Ward identity which, besides the Slavnov and the other identities, has been shown to be free of anomalies. As a second step, one has proceeded to the calculation of the most general local counterterm of the model (3.18), compatible with those symmetry constraints.

The topological vector supersymmetry deminishes the number of potential UV divergences much more drastically than in [9] where the constraint of the vector symmetry could not be imposed. Nevertheless, one ends up with a set of BRS-trivial monomials related to the anomalous dimensions of the model, whose coefficients may be determined by means of Feynman diagrams. The conclusion is that the cohomological nature of the model remains unaltered at the quantum level.

\section{Acknowledgements}

We would like to thank Silvio P. Sorella for many useful comments and suggestions. M.W.O. is grateful to the "Fonds zur Förderung der Wissenschaftlichen Forschung" for a Lise Meitner grant.

\section{Appendix A}

In this appendix we show that there is no loss of generality if one assumes that the local polynomial $\Delta$, defined in eq.(3.13),

$$
\widetilde{\Sigma}=\mathcal{B}_{\Sigma} \Delta
$$

equally obeys the constraints given by the fourth eq. of (3.15) and eqs. (3.16) which are originally verified by the counterterm $\widetilde{\Sigma}$. In other words $\Delta$ can be taken as a rigid invariant object (i.e. a trace in the adjoint representation of the gauge group $\mathbf{G}$ ) satisfying the set of gauge fixing and ghost conditions obtained previously.

We begin by analyzing the rigid gauge invariance $\mathcal{R}^{a}$. As a consequence of its $\mathcal{R}^{a}$ invariance $\widetilde{\Sigma}$ is also invariant under the action of the quadratic Casimir operator, i.e.

$$
\mathcal{R}^{2} \widetilde{\Sigma} \equiv \mathcal{R}^{a} \mathcal{R}^{a} \widetilde{\Sigma}=0
$$

Recalling the commutation relation

$$
\left[\mathcal{R}^{a}, \mathcal{B}_{\Sigma}\right]=0
$$

one may set:

$$
\mathcal{B}_{\Sigma} \mathcal{R}^{2} \Delta=0
$$

The local polynomial $\Delta$ can be split up in two parts:

$$
\Delta=\Delta^{\natural}+\Delta^{b}
$$


where $\Delta^{\natural}$ is invariant and $\Delta^{b}$ is non-invariant under rigid transformations. The noninvariant part decomposes in terms of the eigenvalues of $\mathcal{R}^{2}$ :

$$
\Delta^{b}=\sum_{l=1}^{\bar{l}} \Delta_{l}
$$

with

$$
\mathcal{R}^{2} \Delta_{l}=c_{l} \Delta_{l}
$$

The eigenvalues $c_{l}$ are strictly positive constants, the generators being represented by hermitian matrices. Equation (A.4) can then be rewritten as follows:

$$
\sum_{l=1}^{\bar{l}} c_{l} \mathcal{B}_{\Sigma} \Delta_{l}=0
$$

Since $\mathcal{B}_{\Sigma}$ commutes with the rigid transformations and the $\Delta_{l}$ are independent of each other, the unique solution to (A.8) is

$$
\mathcal{B}_{\Sigma} \Delta_{l}=0, \quad \forall l \geq 1
$$

so that

$$
\mathcal{B}_{\Sigma} \Delta^{b}=0
$$

Hence, the non-invariant part $\Delta^{b}$ is $\mathcal{B}_{\Sigma}$-invariant, giving no contribution to $\widetilde{\Sigma}$.

We now discuss the extension to $\Delta$, of the validity of the gauge fixing and ghost conditions (3.16). The formers read as:

$$
\frac{\delta \widetilde{\Sigma}}{\delta b^{a}}=\frac{\delta \widetilde{\Sigma}}{\delta \eta^{a}}=0
$$

and, by using the redefinitions

$$
\begin{aligned}
\hat{\Omega}_{\mu}^{a} & =\Omega_{\mu}^{a}-\partial_{\mu} \bar{c}^{a}, \\
\hat{\tau}_{\mu}^{a} & =\tau_{\mu}^{a}-\partial_{\mu} \bar{\varphi}^{a},
\end{aligned}
$$

one may rewrite the ghost equations as

$$
\frac{\delta \widetilde{\Sigma}}{\delta \bar{c}^{a}}=\frac{\delta \widetilde{\Sigma}}{\delta \bar{\varphi}^{a}}=0
$$

Now, we introduce the filtration $F$ :

$$
F=\int d^{4} x\left(b^{a} \frac{\delta}{\delta b^{a}}+\bar{c}^{a} \frac{\delta}{\delta \bar{c}^{a}}+\eta^{a} \frac{\delta}{\delta \eta^{a}}+\bar{\varphi}^{a} \frac{\delta}{\delta \bar{\varphi}^{a}}\right)=\left\{\mathcal{B}_{\Sigma}, \bar{c}^{a} \frac{\delta}{\delta b^{a}}+\bar{\varphi}^{a} \frac{\delta}{\delta \eta^{a}}\right\}
$$

and we notice that it commutes with the Slavnov operator,

$$
\left[F, \mathcal{B}_{\Sigma}\right]=0
$$

One has to observe that $F$ will induce a separation on $\Delta$ :

$$
\Delta=\sum_{m=0}^{\bar{m}} \Delta^{(m)}
$$


with

$$
F \Delta^{(m)}=m \Delta^{(m)}
$$

From eq.(A.1) one can write the counterterm as:

$$
\tilde{\Sigma}=\mathcal{B}_{\Sigma}\left(\Delta^{(0)}+\sum_{m=1}^{\bar{m}} \Delta^{(m)}\right)
$$

and, as a consequence of (A.11) and (A.14), we have:

$$
F \widetilde{\Sigma}=0 .
$$

By combining eqs.(A.19) and (A.20), we get:

$$
\sum_{m=1}^{\bar{m}} m \mathcal{B}_{\Sigma} \Delta^{(m)}=0 .
$$

Since the sectors $\Delta^{(m)}$ remain independent under the action of $\mathcal{B}_{\Sigma}$, there is only one possible solution to (A.21), namely

$$
\mathcal{B}_{\Sigma} \Delta^{(m)}=0 \quad \forall m \geq 1
$$

Therefore, we see that all terms $\Delta^{(m)}$ with weights greater than zero play no role in the construction of $\widetilde{\Sigma}$.

To summarize, we have obtained the following result :

$$
\tilde{\Sigma}=\mathcal{B}_{\Sigma} \Delta
$$

with $\Delta$ obeying the constraints given by the rigid invariance, the gauge condition and the ghost equation.

\section{Appendix B}

This appendix contains a detailed description of the use of the antighost equation and of the $\mathcal{F}$-Ward identity as constraints on the evaluation of $\widetilde{\Sigma}$ (see the first two eqs. of (3.15)).

The study of this constraints is greatly simplified if one performs the following field redefinitions t:

$$
\begin{aligned}
\hat{\psi}_{\mu}^{a} & =\psi_{\mu}^{a}+f^{a b c} c^{b} A_{\mu}^{c}, \\
\hat{\Omega}_{\mu}^{a} & =\Omega_{\mu}^{a}+f^{a b c} c^{b} \tau_{\mu}^{c}, \\
\hat{\varphi}^{a} & =\varphi^{a}+\frac{1}{2} f^{a b c} c^{b} c^{c}, \\
\hat{L}^{a} & =L^{a}+f^{a b c} c^{b} D^{c} .
\end{aligned}
$$

\footnotetext{
${ }^{4}$ Here $\Omega_{\mu}$ and $\tau_{\mu}$ stand for the redefined variables of (A.12) and (A.13).
} 
Thereby, in terms of these shifted variables, the first two conditions (3.15) are replaced by:

$$
\int d^{4} x \frac{\delta \widetilde{\Sigma}}{\delta \hat{\varphi}^{a}}=\int d^{4} x \frac{\delta \widetilde{\Sigma}}{\delta c^{a}}=0 .
$$

Now, in order to avoid ambiguities, it behooves us to translate the conditions (B.5) above to a non-integrated level. To do this, we properly substitute functional derivatives by ordinary ones, introducing a prescription in order to handle with $\widetilde{\Sigma}$ and $\Delta$ at the level of their integrands.

The general structure of the local counterterm $\widetilde{\Sigma}$ to be understood here is:

$$
\widetilde{\Sigma}=\sum_{k=1}^{\bar{k}} \int d^{4} x M_{k}(c, \partial c, \ldots ; \hat{\varphi}, \partial \hat{\varphi}, \ldots) X_{k}
$$

where $M_{k}$ is a monomial depending on $c^{a}, \hat{\varphi}^{a}$ and their space-time derivatives; $X_{k}$ is another monomial built up from all other fields and their derivatives. The associated integrand $\tilde{\omega}$ of $\widetilde{\Sigma}$ is then defined to be:

$$
\tilde{\omega}=\sum_{k=1}^{\bar{k}} \tilde{\omega}_{k}
$$

with,

$$
\tilde{\omega}_{k}=\left\{\begin{array}{ccc}
M_{k} X_{k} & \text { if } \quad X_{k} \neq \partial Y_{k} \\
-\partial M_{k} Y_{k} & \text { if } \quad X_{k}=\partial Y_{k}
\end{array}\right.
$$

and, repeatedly, if $X_{k}=\partial \partial Y_{k}$ then, $\tilde{\omega}_{k}=\partial \partial M_{k} Y_{k}$, and so on. With this prescription in mind, one is allowed to rewrite expression (A.1) in a unique way:

$$
\tilde{\omega}=\mathcal{B}_{\Sigma} \omega
$$

$\omega$ being the integrand of $\Delta$, defined according to the same rule. That prescription (B.8) is unique, can be easily seen from the fact that:

$$
\widetilde{\Sigma}=\int d^{4} x \tilde{\omega}=0 \quad \Rightarrow \quad \tilde{\omega}=0 .
$$

Indeed, one has in general the following:

$$
\begin{aligned}
\widetilde{\Sigma}=0 \Rightarrow \quad \widetilde{\Sigma} & =\int d^{4} x \partial\left(\sum_{k=1}^{\bar{k}} M_{k}(c, \ldots ; \hat{\varphi}, \ldots) X_{k}\right) \\
& =\int d^{4} x\left(\sum_{k=1}^{\bar{k}} \partial M_{k} X_{k}+\sum_{k=1}^{\bar{k}} M_{k} \partial X_{k}\right)
\end{aligned}
$$

where $X_{k}$ may be a total space-time derivative or not. If yes, i.e. if

$$
X_{k}=\partial Y_{k},
$$

then, with (B.8)

$$
\tilde{\omega}_{k}=-\partial \partial M_{k} Y_{k}+\partial \partial M_{k} Y_{k}=0 .
$$


On the other hand, if $X_{k}$ is non-differentiated, one has again:

$$
\tilde{\omega}_{k}=\partial M_{k} X_{k}-\partial M_{k} X_{k}=0
$$

At the level of the integrands, the constraints (B.5) read

$$
\frac{\partial \tilde{\omega}}{\partial \hat{\varphi}^{a}}=\frac{\partial \tilde{\omega}}{\partial c^{a}}=0
$$

We now define the filtration,

$$
\stackrel{\circ}{F}=c^{a} \frac{\partial}{\partial c^{a}}+\hat{\varphi}^{a} \frac{\partial}{\partial \hat{\varphi}^{a}}
$$

which commutes with $\mathcal{B}_{\Sigma}$ :

$$
\left[\stackrel{\circ}{F}, \mathcal{B}_{\Sigma}\right]=0
$$

From $($ B.10) follows

$$
\stackrel{\circ}{F} \tilde{\omega}=0 .
$$

Since the filtration induces a separation on $\omega$, one can write eq. (B.9) as:

$$
\tilde{\omega}=\mathcal{B}_{\Sigma}\left(\omega^{(0)}+\sum_{p=1}^{\bar{p}} \omega^{(p)}\right) .
$$

Applying the filtration on both sides of (B.14) implies

$$
\sum_{p=1}^{\bar{p}} p \mathcal{B}_{\Sigma} \omega^{(p)}=0
$$

with one single solution,

$$
\mathcal{B}_{\Sigma} \omega^{(p)}=0 \quad \forall p \geq 1
$$

We conclude then that

$$
\tilde{\omega}=\mathcal{B}_{\Sigma} \omega^{(0)} .
$$

Hence, coming back to the integrated level, one can disregard the contributions stemming from monomials in $\Delta$ which depend on $c^{a}$ and $\hat{\varphi}^{a}$. Thus

$$
\widetilde{\Sigma}=\mathcal{B}_{\Sigma} \Delta^{(0)},
$$

$\Delta^{(0)}$ being independent of $c^{a}$ and $\hat{\varphi}^{a}$ obeys the constraints (B.5).

\section{References}

[1] E. Witten, Comm. Math. Phys. 117 (1988) 353.

[2] S.K. Donaldson, J. Diff. Geom. 18(1983)269; 26(1987)397; Topology 29(1990)257.

[3] D. Birmingham, M. Blau, M. Rakowski and G. Thompson, Phys. Rep. 209(1991)129. 
[4] R. Brooks, D. Montano and J. Sonnenschein, Phys. Lett. B214 (1988) 91.

[5] J.H. Horne, Nucl. Phys. B318 (1989) 22.

[6] D. Birmingham, M. Rakowski and G. Thompson, Nucl. Phys. B329 (1990) 83.

[7] D. Birmingham, M. Blau and G. Thompson, Int. J. Mod. Phys. A5 (1990) 4721.

[8] J.O. Madsen, Mod. Phys. Lett. A5 (1990) 1013.

[9] M. Werneck de Oliveira, Phys. Lett. B307 (1993) 347.

[10] C. Becchi, A. Rouet and R. Stora, Comm. Math. Phys. 42(1975)127; Ann. Phys. (N.Y.) 98 (1976) 287.

[11] C. Becchi, "The Renormalization of Gauge Theories", in the Proceedings of Les Houches Summer School 1983, eds. B.S. De Witt and R. Stora, North Holland, Amsterdam, 1984.

[12] O. Piguet and A. Rouet, Phys. Reports 76 (1981) 1.

[13] F. Delduc, F. Gieres and S.P. Sorella, Phys. Lett. B225 (1989) 367.

[14] D. Birmingham and M. Rakowski, Phys. Lett. B272(1991) 217.

[15] N. Maggiore and S.P. Sorella, Nucl. Phys. B377 (1992) 236.

[16] M. Schweda, S.P. Sorella and M. Werneck de Oliveira, Phys. Lett. B315 (1993) 93.

[17] L. Baulieu and I.M. Singer, Nucl. Phys. Proc. Suppl. 5B(1988)12.

[18] J.M.F. Labastida and M. Pernici, Phys. Lett. B212 (1988) 56.

[19] S. Ouvry, R. Stora and P. Van Baal, Phys. Lett. B220 (1989) 159.

[20] A. Blasi, O. Piguet and S.P. Sorella, Nucl. Phys. B356 (1991) 154.

[21] C. Becchi, A. Blasi, G. Bonneau, R. Collina and F. Delduc, Comm. Math. Phys. 120 (1988) 121.

[22] J.A. Dixon, "Cohomology and Renormalization of Gauge Theories", preprints Imperial College, 1977; Comm. Math. Phys. 139 (1991) 495.

[23] O. Piguet and K. Sibold, "Renormalized Supersymmetry", Birkhäuser, Boston 1986.

[24] F. Brandt, N. Dragon and M. Kreuzer, Phys. Lett. B231 (1989) 263. 